\title{
RHEOLOGIC STUDIES ON SYNOVIAL FLUID
}

\author{
By MILTON G. LEVINE AND DAVID H. KLING
}

(From the Boyar-Kling Arthritis Clinic, Los Angeles, Calif.)

(Submitted for publication March 28, 1956; accepted September 5, 1956)

The relative viscosity of normal and pathological synovial fluids fluctuates over a wide range, and has been studied for a considerable period. The older literature is covered by Kling (1), and the more recent by Ropes and Bauer (2) and by Sundblad (3). Early investigators established, by precipitation reactions with acetic acid, that the high viscosity was due to a "mucin," assumed to be a combination of protein and carbohydrate. In 1939, Meyer, Smyth, and Dawson (4) identified the polysaccharide as hyaluronic acid, and found it to be responsible for the high viscosity.

Further studies revealed that solutions of hyaluronic acid in common with other macromolecular substances exhibited non-Newtonian viscosity. Therefore, relative viscosity, heretofore the only viscosity factor determined, was no longer sufficient to characterize the rheological behavior of synovial fluid. Other types were investigated, including intrinsic and anomalous viscosity.

In the following studies we describe a simple capillary viscometer which can be used to measure relative, intrinsic and anomalous viscosity.

Results are presented of viscosity determinations in synovial fluids from normal joints of animals and pathological effusions from various types of human arthritis.

\section{DESCRIPTION OF VISCOMETER}

Our instrument (Figure 1) is a modification of the capillary viscometer described by Mann (5) for measuring plasma fluidity. The Mann, like the standard Ostwald viscometer, cannot be used conveniently to measure anomalous viscosity because it is constructed to operate at a fixed stress. By substituting a graduated pipette for the volumetric pipette suggested by Mann we have been able to measure the anomalous viscosity under variable stress.

In most instances we used a one-ml. pipette, graduated in $0.1 \mathrm{ml}$. The length of the pipette from zero point to tip was about $23 \mathrm{~cm}$. Other calibers and lengths of the pipette may be selected. The capillary consisted of a spinal or hypodermic needle about $10 \mathrm{~cm}$. in length attached to the delivery end of the pipette. The length and gauge of the needle may be varied. A 20 gauge is suitable for most joint fluids; a 22 gauge is preferable for fluids of low viscosity, and an 18 gauge for highly viscid fluids.

The attachment of the needle to the pipette is made water-tight by a rubber sleeve, or by a cement seal, or the delivery end of the pipette may be ground so that it fits snugly into the hilt of the needle.

The viscometer is filled by suction above the zero mark, and the upper opening of the pipette is kept closed with a finger. The pipette is placed in a clamp holder and immersed in a constant temperature bath until the hilt of the hypodermic needle meets the water level. The measurement is started by releasing the finger, and the rate of flow is timed between two selected points on the pipette, either by means of two stop watches or an electronic recorder. Readings are made at close to room temperature. The water level is not appreciably altered by the small amount of synovial fluid which flows into the bath. The metal capillary adjusts almost instantaneously to the temperature of the water bath. Differences between the temperature of the synovial fluid in the pipette and the temperature of the water bath have only a slight effect on the efflux time as shown in the following experiment :

With the water bath temperature kept constant at $21.5^{\circ}$ $\mathrm{C}$ the temperature of the fluid in the pipette was varied between $17^{\circ} \mathrm{C}$ and $37^{\circ} \mathrm{C}$. At $17^{\circ} \mathrm{C}$ the average efflux time for 10 determinations was 15.4 seconds; at $27^{\circ} \mathrm{C}$ the average time was 15.2 seconds; at $37^{\circ} \mathrm{C}$ the average time was 14.9 seconds. In this severe test of the effect of the temperature of the fluid in the pipette on the efflux time the variations were minor over a temperature range of $20^{\circ} \mathrm{C}$. In actual practice the temperature of the fluid and the temperature of the water bath are kept close together, so that the temperature error is negligible. The experiment quoted was representative of three which gave similar results.

If the rate of shear is measured for every $0.1 \mathrm{ml}$. of fluid volume, from zero to $0.9 \mathrm{ml}$., the first pressure will be an average of : $h_{1}$-the distance from the exit point of the capillary to the zero point on the pipette, and $h_{2}-$ the distance from the exit point of the capillary to $0.1-\mathrm{ml}$. mark on the pipette.

With every drop of $0.1 \mathrm{ml}$., which corresponds to about $2 \mathrm{~cm}$. in length on the pipette, there is a corresponding decrease in the average pressure head. The unit volume may be varied as well as the pressure head. This may be done by changing the distance between $h_{1}$ and $h_{2}$.

A constant temperature is maintained in the bath with a thermostatically controlled heating unit. It is best not to use a mechanical stirrer, which may disturb the water level and increase turbulence at the end of the capillary. 


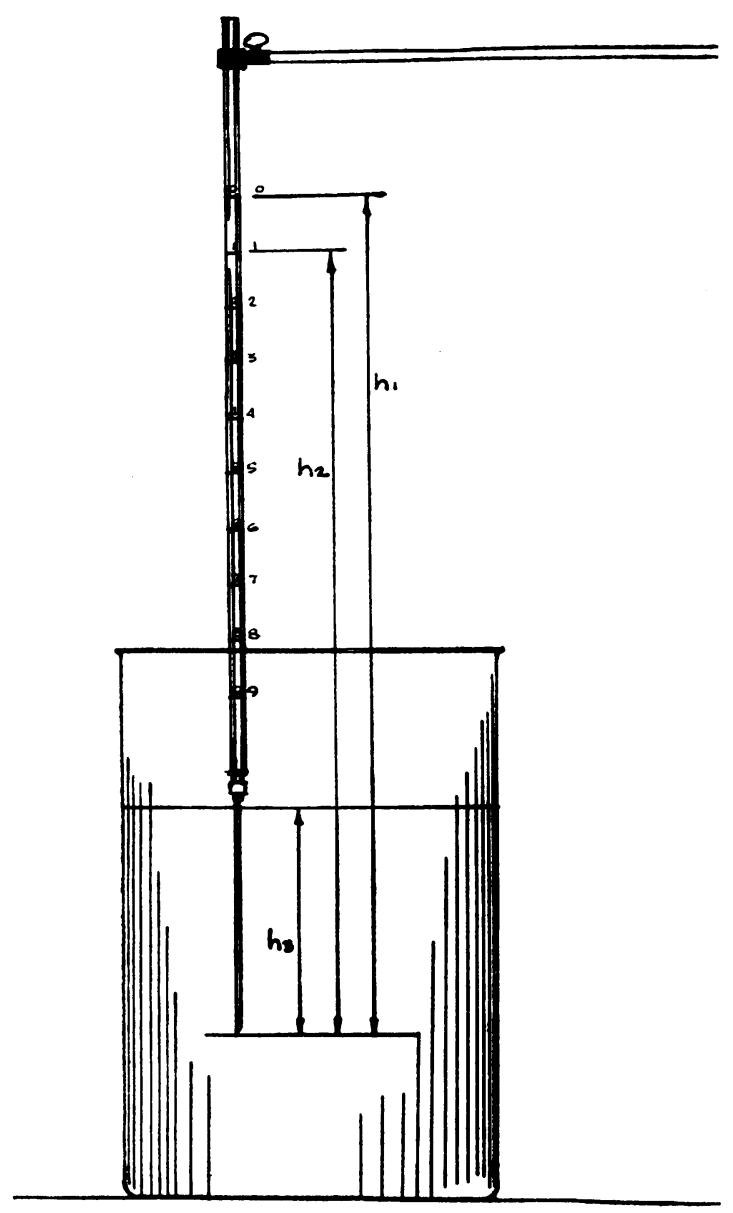

Fig. 1. Simple Capillary Viscometer

F If the efflux time is determined between the zero point and the 0.1-ml. mark on the graduated pipette, the average pressure head ( $p$ ) for a fluid of density (d) will be :

$$
p=d\left[\frac{\left(h_{1}+h_{2}\right)}{2}\right]-h_{3} .
$$

A gallon jar filled with water of the desired temperature will keep a constant temperature for several hours.

Repeated determinations of the efflux time vary only within 0.3 of a second. Proper cleaning is of great importance because dust or other particles may partially obstruct the pipette and needle and change the efflux time. In contrast to most capillary viscometers, cleaning is very easily done by attaching the pipette to a suction pump and rinsing with distilled water and alcohol, and drying with ether. The metal capillary is unbreakable. The kinetic energy correction is small in this viscometer, less than 1 per cent, and has been neglected in the calculations.

In the determination of relative viscosity in any single viscometer, all factors are constant except the efflux time and density. To obtain the relative viscosity it is necessary to compare the flow time of the solution with the flow time of the solvent, which in the case of synovial fluid is water. In our material the density of the synovial fluids varied from 1.012 to 1.025 , with an average of 1.019 , and could be neglected.

Based on Poiseuille's formula, the relative viscosity of synovial fluids measured by our viscometer is expressed as : $\eta$ rel $=\frac{d t s}{t w}$. Where $t s$ is the time of efflux of the synovial fluid, $t w$ the flow time for water, and $d$ is the density of synovial fluid.

Any of a number of pressure heads may be selected to determine relative viscosity. We used an average hydrostatic pressure of approximately $12.8 \mathrm{~cm}$. of water, which corresponded to the $0.5-\mathrm{ml}$. and $0.6-\mathrm{ml}$. gradation on the pipette used in these studies. The efflux time of $0.1-\mathrm{ml}$. water was 2.8 seconds. The length of the capillary was $9.9 \mathrm{~cm}$., the diameter $0.29 \mathrm{~mm}$. The length of the pipette from tip to zero point was $23.2 \mathrm{~cm}$., the bore was $3 \mathrm{~mm}$., and the temperature of the water bath was $29^{\circ} \mathrm{C}$.

\section{MATERIAL AND METHODS}

Synovial fluid from normal human joints was not available to us. Therefore, synovial fluid was aspirated from the carpal joints of horses and cattle obtained from the packing house immediately after slaughter. After aspiration, the joints were opened. Fluid was used only from joints with grossly normal articular cartilage and synovial membrane. Roentgenograms showed the articular surfaces to be intact. Microscopic sections of the synovial membrane of these horses and cattle were normal, except that in the former, slight edema and hyperemia were frequently present. Pathological effusions were aspirated from the knee joints of patients with rheumatoid arthritis, osteoarthritis (degenerative) and chronic traumatic synovitis.

Viscosity determinations were done after the fluid was freed from suspended cells and other particles by prolonged centrifugation. If the viscosity tests could not be run immediately, the fluid was kept in the refrigerator at $4^{\circ} \mathrm{C}$. No changes of viscosity occurred even after prolonged storage.

The hyaluronic acid concentration was determined by a combination of the turbidimetric methods described by Meyer (6) and by Schmith and Faber (7). Ragan and Meyer (8) found that the turbidimetric method is satisfactory for the determination of hyaluronic acid in synovial fluid. The method is based on the fact that partially depolymerized hyaluronic acid at a pH of about 4.2 produces a colloidal suspension in the presence of serum protein (9). Synovial fluid was treated with a minimal amount of hyaluronidase to assure uniform turbidity. The amount used was determined by titration. Serum reagent was prepared by the method of Schmith and Faber (7), frozen, thawed out before use, and heated at $99^{\circ} \mathrm{C}$ for ten minutes as recommended by Tolksdorf, McCready, McCullagh, and Schwenk (10).

The steps of the determination were as follows:

In a test tube

Add $0.1 \mathrm{ml}$. of synovial fluid to $0.8 \mathrm{ml}$. of 0.85 per cent sodium chloride 
TABLE I

Relative viscosity of synovial fluid*

\begin{tabular}{|c|c|c|c|c|c|c|c|}
\hline \multirow[b]{2}{*}{ Species } & \multirow[b]{2}{*}{ Joint } & \multirow[b]{2}{*}{ Diagnosis } & \multirow[b]{2}{*}{ No. } & \multicolumn{2}{|c|}{ Volume, ml. } & \multicolumn{2}{|c|}{ Viscosity } \\
\hline & & & & Range & Average & Range & Average \\
\hline $\begin{array}{l}\text { Cattle } \\
\text { Horse } \\
\text { Human }\end{array}$ & $\begin{array}{l}\text { Carpal } \dagger \\
\text { Carpal } \\
\text { Knee }\end{array}$ & $\begin{array}{l}\text { Normal } \\
\text { Normal } \\
\text { Osteo- } \\
\text { arthritis }\end{array}$ & $\begin{array}{l}40 \\
25 \\
87\end{array}$ & $\begin{array}{l}2-10 \\
2-12 \\
1-120\end{array}$ & $\begin{array}{r}5 . \\
6 . \\
32 .\end{array}$ & $\begin{array}{c}9-10,300 \\
4.5-119 \\
10-2,830\end{array}$ & $\begin{array}{r}929 \\
16 \\
106\end{array}$ \\
\hline Human & Knee & $\begin{array}{c}\text { Rheumatoid } \\
\text { arthritis }\end{array}$ & 168 & $2-200$ & 29. & $2-83$ & 18 \\
\hline Human & Knee & $\begin{array}{l}\text { Chronic } \\
\text { traumatic } \\
\text { synovitis }\end{array}$ & 32 & $4-90$ & 32. & $2-68$ & 20 \\
\hline
\end{tabular}

* The relative viscosity of some pathological effusions was determined by Mann and other capillary viscometers which gave values in the range of our instrument.

$\dagger$ This is a composite articulation of three joints in the foreleg: 1) the radio-carpal joint; 2) the intercarpal joint; 3) the carpo-metacarpal joint. These joints are equivalent to the wrist joint of humans, but are usually referred to as knee joints of animals. The fluids were obtained from the two proximal joints.

Add $0.1 \mathrm{ml}$. of hyaluronidase solution previously titrated Incubate at $37^{\circ} \mathrm{C}$ for thirty minutes

Add $1 \mathrm{ml}$. of buffer solution $\mathrm{pH} 6(0.1 \mathrm{M}$ sodium acetate and $0.15 \mathrm{M}$ sodium chloride)

Add $8 \mathrm{ml}$. of diluted serum reagent made by diluting serum reagent with $0.5 \mathrm{M}$ sodium acetate buffer $\mathrm{pH} 4.2$ dilution of $1: 4$

Incubate at $30^{\circ} \mathrm{C}$ for thirty minutes and read the turbidity in a Coleman spectrophotometer at $580 \mathrm{~m} \mu$

Serum reagent is prepared as follows :

Horse serum is diluted $1: 10$ with $0.5 \mathrm{M}$ sodium acetate buffer $\mathrm{pH}$ 4.2. The $\mathrm{pH}$ is adjusted to 3.1 by the addition of $4 \mathrm{~N} \mathrm{HCl}$.

The mixture is frozen. Before use it is thawed out and heated at $99^{\circ} \mathrm{C}$ for ten minutes and then diluted $1: 4$ with $0.5 \mathrm{M}$ sodium acetate buffer.

The turbidimetric method gave reproducible curves within 5 per cent. The turbidities disappeared after exposure to an excess of testicular and streptococcal hyaluronidase. The latter attacks only hyaluronic acid; therefore, this is evidence that hyaluronic acid and no other mucopolysaccharide was determined by the turbidimetric method. Furthermore, electrophoretic patterns of normal and pathological synovial fluids have shown boundaries characteristic only for mucopolysaccharides of a mobility in the range of hyaluronic acid (11).

Total and differential cell counts, icterus index, $\mathrm{pH}$, total protein, albumin and globulin, uric acid, bacterial cultures, guinea pig inoculations, serological and other tests were performed on part of the material.

\section{RESULTS}

Relative viscosity was determined in 65 normal fluids from animals and 287 pathological effusions (Table I). The highest values, and also the widest range of viscosity, were obtained from the normal fluids of the carpal joints of cattle. Fluid from the carpal joints of horses showed a markedly narrower range and a much lower average relative viscosity. Sundblad (3) found very low relative viscosities from 3 to 29 in 10 fluids from normal astragalotibial joints of horses. He ascribed the low values to dilution with plasma as evidenced by large volumes ranging from 5 to $40 \mathrm{ml}$. However, in our material the volume in both cattle and horse joints was fairly small and the range was almost identical ( 2 to 10 and 2 to $12 \mathrm{ml}$.).

Several factors must be considered to explain the discrepancy between the relative viscosity of the fluids from normal carpal joints of cattle and horses. First, there is an age difference. The age of cattle ranged from 10 months to 3 years, whereas the horses averaged 8 years. Second, edema and hyperemia in some of the synovial membranes of the horses have led to exudation. Even small dilutions of 5 per cent to 10 per cent can cause an enormous fall in relative viscosity, as first pointed out by Schneider (12). This is especially true of normal fluids with highly polymerized hyaluronic acid. Other contributing factors will be taken up later in the discussion.

The relative viscosity of the pathological effusions from osteoarthritis had the widest range and the highest average values. On the other hand, the effusions in rheumatoid arthritis and chronic traumatic synovitis had significantly small ranges and lower average values.

\section{Intrinsic viscosity}

Intrinsic viscosity $[\eta]$ is the limiting value of the ratio $\frac{(\eta s p)}{c}$ at zero concentration. For the 
calculation of the intrinsic viscosity, we used the derivation of Huggin's formula originally suggested by Schulz and Blaschke (13) and employed by Sundblad (3) for determining the viscosity of synovial fluid :

$$
[\eta]=\frac{\frac{(\eta s p)}{c}}{1+k^{\prime}(\eta s p)} .
$$

The specific viscosity $(\eta s p)$ is equal to the relative viscosity minus one. In this formula $k^{\prime}$ is approximately constant for members of a homologous series of polymers in a given solvent. It can be found experimentally by first determining intrinsic viscosity by extrapolation. Sundblad (3) reported the average value for $k^{\prime}$ to be about 0.16 for hyaluronic acid from normal and pathological fluid. Substituting 0.16 for $k^{\prime}$ and knowing the concentration, the intrinsic viscosity may be determined merely by obtaining the specific viscosity. Sundblad found this to be true for specific viscosities of from 0.3 to 15.0 .

Thus it is possible to dilute fluids to an arbitrary specific viscosity below 15 , and by determining the concentration of hyaluronic acid at that dilution, the intrinsic viscosity may be derived from Sundblad's formula.

The results of hyaluronic acid determinations and the intrinsic viscosity on 54 animal and 56 pathological effusions are given in Table II. The values for both intrinsic viscosity and hyaluronic acid concentration fluctuate in a considerable range. The highest average intrinsic viscosity (43.8) occurred in synovial fluid from normal carpal joints of cattle. The average values from the same joint of the horses were lower (36.1). For the pathological effusions, the average intrinsic viscosities in osteoarthritis (35.9) and in traumatic synovitis
(33.3) were higher than in rheumatoid arthritis (29.1). Since the intrinsic viscosity reflects the mean polymerization, it is observed that the most highly polymerized hyaluronic acid was found in the normal synovial fluid from cattle, and the lowest in effusions from rheumatoid arthritis.

The fluids of the normal carpal joints of the cattle show a high average hyaluronic acid concentration (200 mg. per cent), while in the same joint of the horses the values were lower $(136 \mathrm{mg}$. per cent). Sundblad (3) gives the following values for 11 normal calves: volume one to three $\mathrm{ml}$., hyaluronic acid concentration $193 \mathrm{mg}$. per cent. This is in the range of our own values of the joints in young cattle. Only in one ox did Sundblad find a low value of $45 \mathrm{mg}$. per cent. The volume of the fluid here was high, namely, $30 \mathrm{ml}$.

In osteoarthritis the average concentration of hyaluronic acid ( $214 \mathrm{mg}$. per cent) is greater than in rheumatoid arthritis and chronic traumatic synovitis (158 mg. per cent and $148 \mathrm{mg}$. per cent, respectively). Higher concentrations of hyaluronic acid in osteoarthritis than in rheumatoid arthritis were also found by Fletcher, Jacobs, and Markham (14). Sundblad (3), on the other hand, reported the concentration of hyaluronic acid in osteoarthritis to be similar to that in rheumatoid arthritis.

\section{Anomalous viscosity}

Anomalous viscosity of synovial fluid has been investigated with the Couette viscometer by Fessler, Ogston, and Stanier (15), or with the horizontal capillary viscometer with or without manostat controlled pressure by Johnston (16), Scott Blair, Williams, Fletcher, and Markham (17), and Sundblad (3). Ostwald and Auerbach (18) have shown that anomaly can be meas-

TABLE II

Intrinsic viscosity and hyaluronic acid concentration of synovial fluid

\begin{tabular}{llllll}
\hline \hline Species & Joint & Diagnosis & No. & $\begin{array}{c}\text { Intrinsic } \\
\text { viscosity }\end{array}$ & $\begin{array}{c}\text { Concentration } \\
\text { hyaluronic acid } \\
\text { mg. \% }\end{array}$ \\
\hline Cattle & Carpal & Normal & 30 & $43.8 \pm 3.9 *$ & $200 \pm 57$ \\
Horse & Carpal & Normal & 24 & $36.1 \pm 7.8$ & $136 \pm 77$ \\
Human & Knee & Osteoarthritis & 16 & $35.9 \pm 8.7$ & $214 \pm 91$ \\
Human & Knee & Rheumatoid & 32 & $29.1 \pm 5.4$ & $158 \pm 60$ \\
Human & Knee & Chronic & $\mathbf{8}$ & $33.3 \pm 8.4$ & $148 \pm 86$ \\
& & traumatic & & & \\
& & synovitis & & &
\end{tabular}

* Standard deviation. 


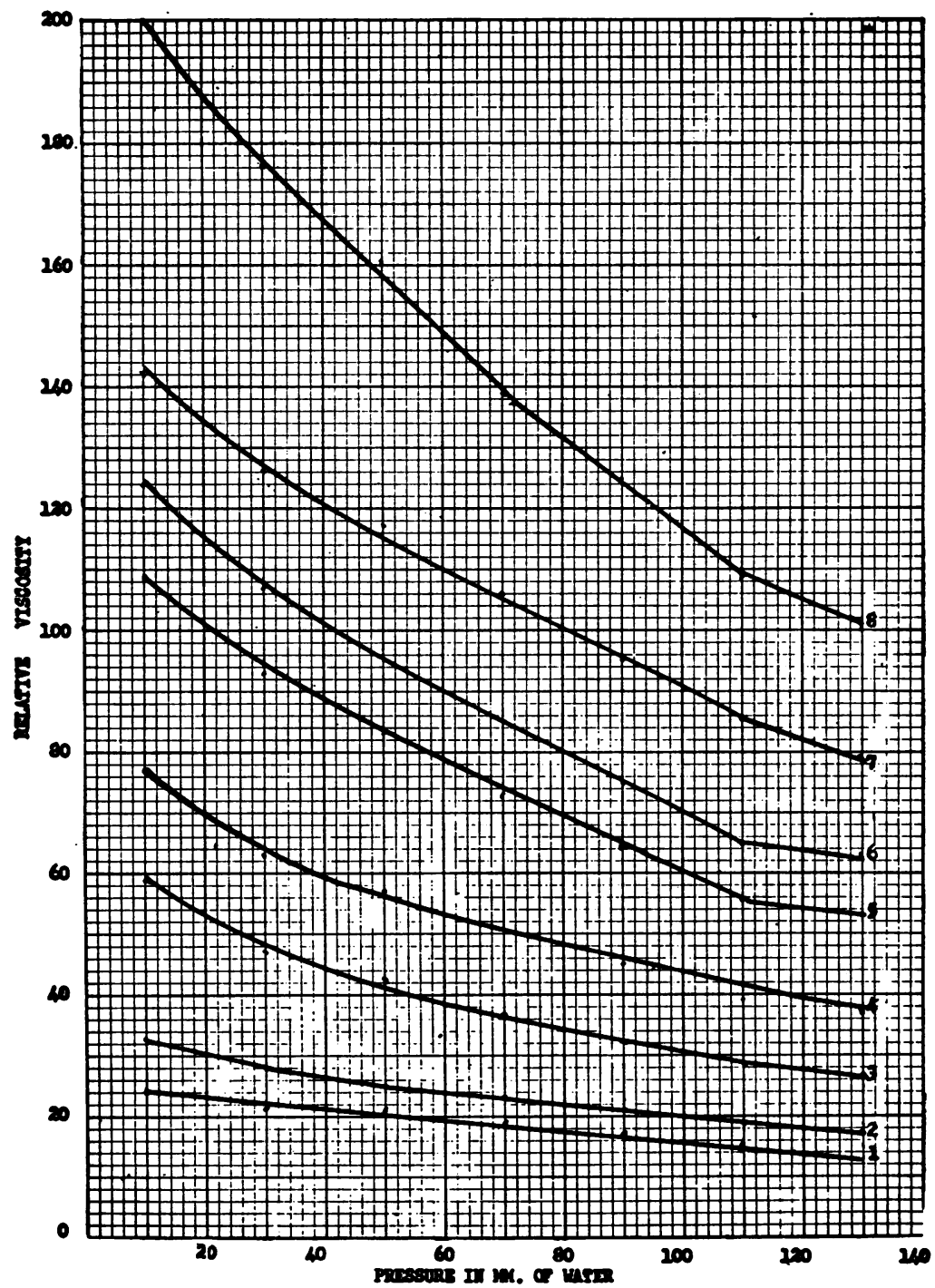

Fig. 2. Anomalous Viscosity

The relative viscosity varies with the hydrostatic pressure forcing the liquid through the capillary. The lower three curves are of synovial fluid from patients with rheumatoid arthritis. The upper five curves are of synovial fluid from patients with osteoarthritis. The curves demonstrate the increase in anomalous viscosity with increasing relative viscosity.

ured accurately in a vertical capillary viscometer, using gravity alone or with added pressure to vary the stress. Zamboni (19) has demonstrated that the peak of anomaly for extracts of "mucin" from synovial fluid and umbilical cord occurs at hydrostatic pressures below $10 \mathrm{~cm}$. of water. The dimensions of both the capillary and the pipette employed in our instrument fall within the range generally regarded as satisfactory for viscosity measurements of hyaluronic acid.
In our viscometer, anomaly is measured by varying the hydrostatic pressure. As the height of the column of fluid falls, the hydrostatic pressure decreases and the relative viscosity increases. The gradations on the pipette of our viscometer correspond to $0.1 \mathrm{ml}$. in volume. For the whole pipette, which holds one ml., there are ten possible points at which the efflux time can be measured. We have read the points corresponding to $0.1 \mathrm{ml}$., $0.2 \mathrm{ml}$., etc., down to $0.9 \mathrm{ml}$. The greatest ac- 
curacy lies in the efflux time readings between the points on the pipette corresponding to $0.3 \mathrm{ml}$. and $0.8 \mathrm{ml}$. If we plot flow time for this series of consecutive points converted into centimeters of wa- ter pressure by measuring the height of the column, the resulting curve is indicative of the rate of increase of the anomalous viscosity (Figure 2). This curve is dependent upon two variables, the

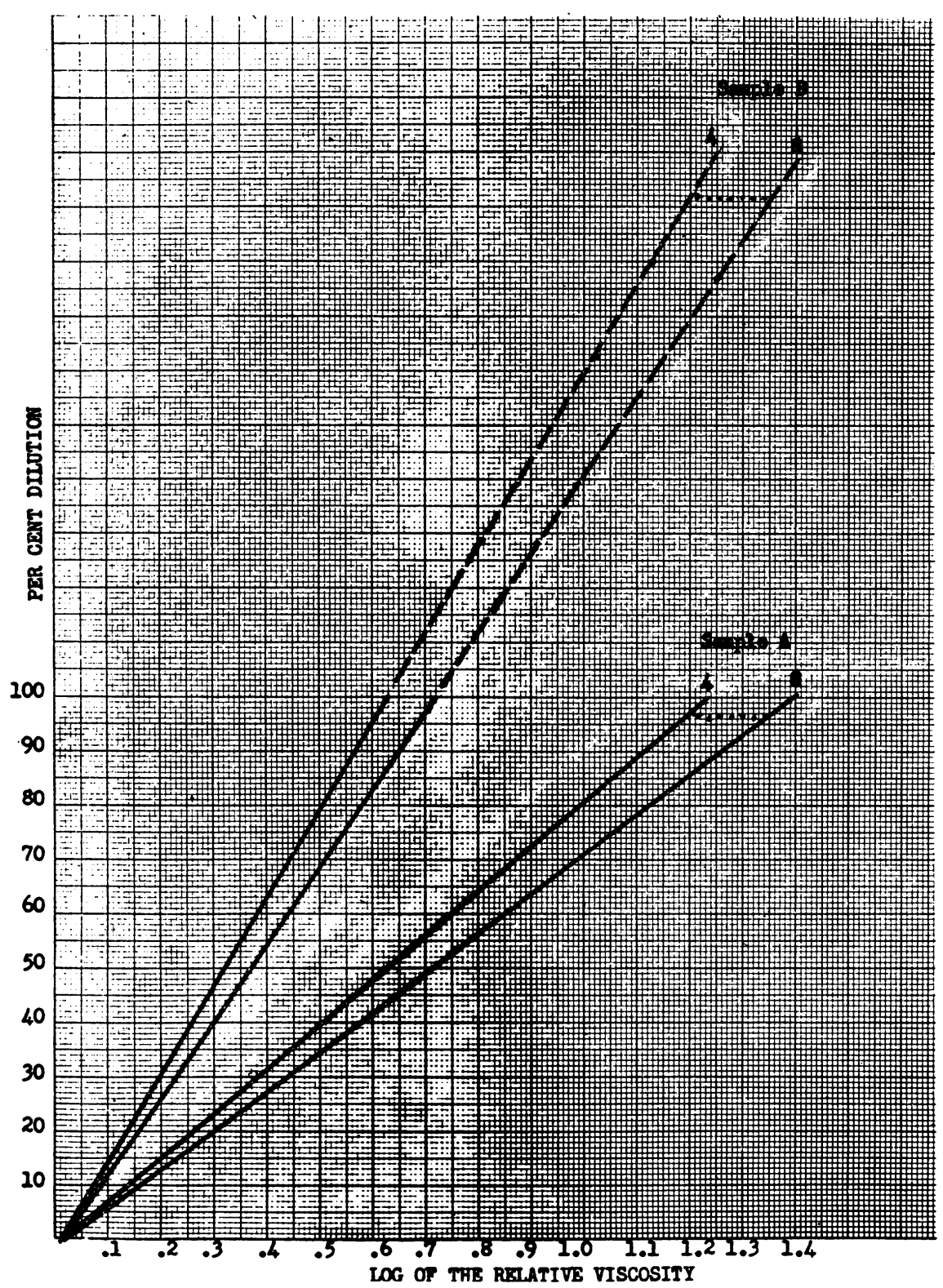

Fig. 3. Curves for Determining the Anomaly Factor

The reference point is the $\log 1.2$ for the relative viscosity at the 4th point of stress (corresponding to a pressure of $170 \mathrm{~mm}$. of water). The dilution which gives this relative viscosity is then found on the curve for the 8th point of stress (corresponding to a pressure of $85 \mathrm{~mm}$. of water). The difference between the logs of the relative viscosities at the two points of stress is the anomaly factor. In the figure the dotted line indicates this difference. Samples " $A$ " and " $B$ " are identical in polymerization, but " $B$ " contains half the concentration of hyaluronic acid of sample "A." Both samples give the same anomaly factor $(0.15)$. 
concentration and polymerization of hyaluronic acid in the synovial fluid. For the anomaly to reflect the degree of polymerization alone, the effect of concentration of hyaluronic acid must be eliminated.

Our method for doing this is based on the fact that the logarithms of the relative viscosities of a series of dilutions of synovial fluid give nearly a straight line when plotted against per cent dilution. If this is done for two different points of stress on our viscometer, two straight lines result which converge near the point of infinite dilution. We have arbitrarily chosen the logarithm of one particular relative viscosity at one point of stress as a reference point. The increase in the viscosity which occurs at a second point of stress for the same dilution is indicative of the increase in the degree of anomaly. To illustrate that this occurs independent of the concentration, we have tested two samples of fluid (Figure 3 ). One fluid has been diluted to 50 per cent of the concentration of hyaluronic acid of the first. The degree of polymerization is the same. The reference point is the $\log 1.2$ for the relative viscosity at the fourth point of stress which corresponds to an average hydrostatic pressure of approximately $170 \mathrm{~mm}$. of water. In sample " $A$ " this corresponds to a dilution of 97 per cent. For the same dilution at the eighth point of stress with average hydrostatic pressure, corresponding to $85 \mathrm{~mm}$. of water, the logarithm is found to be 1.35 . The difference between the logarithms at the eighth and the fourth points of stress is equal to 0.15 . This we have labelled the anomaly factor.

In sample " $B$ " which has half the concentration of hyaluronic acid of " $A$," the reference point must be the same as for sample "A." In order to obtain this value the curve for the fourth point of stress is extrapolated to cross the reference point $\eta$ rel logarithm 1.2. In like manner, the curve for the eighth point of stress is also extrapolated. At the dilution where the fourth point of stress crosses the line for $\log 1.2$ the corresponding point for the eighth point of stress is $\log 1.35$. The difference, or anomaly factor, again is 0.15 . In other words, regardless of the initial concentration, this method for determining anomalous viscosity gives the same anomaly factor, which then may be considered directly proportional to the degree of polymerization.
It must be stressed that we have arbitrarily chosen the reference point as $\eta$ rel $\log 1.2$. Any other point is just as good, providing it is used consistently. Fluids diluted to a relative viscosity below 20 are preferable for this type of determination because in highly viscid fluids the straight line tends to curve.

The intrinsic viscosity is chiefly an index of mean polymerization of hyaluronic acid. According to Sundblad (3), the degree of anomalous viscosity is more sensitive to the presence of small amounts of high polymer fractions in pathological fluids, whereas in highly degraded fluids the intrinsic viscosity is more reliable.

Table III lists the average anomaly factors for 33 normal fluids from the carpo-metacarpal joints of cattle and horses and 39 effusions from three types of arthritis. The highest values were found in the fluids from the cattle. The fluid from the horses had lower values, although there is an overlap between these two groups.

Of the pathological fluids, osteoarthritis (degenerative) has higher values than rheumatoid arthritis and chronic traumatic synovitis. Here again there is an overlap. The analysis of results indicates higher polymerization of hyaluronic acid in normal synovial fluid from cattle and horses than from pathological human effusions. In osteoarthritis (degenerative) the average degree of polymerization is higher than in rheumatoid arthritis and traumatic synovitis.

\section{DISCUSSION}

With the simple capillary viscometer described, we have determined relative, intrinsic and anomalous viscosity.

Relative viscosity, the simplest and most frequently used parameter, is not only important in

TABLE III

Anomalous viscosity of synovial fluid

\begin{tabular}{|c|c|c|c|c|}
\hline Species & Joint & Diagnosis & No. & $\begin{array}{c}\text { Average } \\
\text { anomaly } \\
\text { factor }\end{array}$ \\
\hline $\begin{array}{l}\text { Cattle } \\
\text { Horse } \\
\text { Human } \\
\text { Human } \\
\text { Human }\end{array}$ & $\begin{array}{l}\text { Carpal } \\
\text { Carpal } \\
\text { Knee } \\
\text { Knee } \\
\text { Knee }\end{array}$ & $\begin{array}{l}\text { Normal } \\
\text { Normal } \\
\text { Osteoarthritis } \\
\text { Rheumatoid } \\
\text { Chronic } \\
\text { traumatic } \\
\text { synovitis }\end{array}$ & $\begin{array}{r}20 \\
13 \\
10 \\
20 \\
9\end{array}$ & $\begin{array}{l}.281 \pm .068^{*} \\
.227 \pm .04 \\
.177 \pm .034 \\
.122 \pm .034 \\
.137 \pm .035\end{array}$ \\
\hline
\end{tabular}

* Standard deviation. 
itself, but is also necessary for the determination of intrinsic and anomalous viscosity. The latter two are primarily used to find the degree of polymerization, the molecular configuration and molecular weight of hyaluronic acid.

The normal synovial fluids from the carpal joints of cattle show high hyaluronic acid concentrations, the highest intrinsic and anomalous viscosity, as well as the highest average relative viscosity with the greatest range. The volumes fluctuated from 2 to $10 \mathrm{ml}$. with an average of $5 \mathrm{ml}$. Offhand, it seems strange that the range of relative viscosities was so great, from 9 to 10,300 , in the fluids from normal cattle joints. However, it is characteristic of highly polymerized hyaluronic acid in synovial fluid that minimal dilution is followed by great decreases in relative viscosity. Similar wide variations in the range of relative viscosity have been reported for normal human synovial fluid.

The synovial fluid from the carpal joints of the horses had a lower degree of polymerization, as evidenced by the lower intrinsic and anomalous viscosity, and a lower range and average relative viscosity, although the volumes of fluid in both species were similar.

The lower relative viscosity of the horse synovial fluid as compared to the cattle fluid is assumed to be due partly to a lower concentration of hyaluronic acid and partly to a lesser degree of polymerization. While both the cattle and horse joints appeared to be grossly normal, the synovial membrane of the horses showed hyperemia and edema on microscopic examination. Age and species differences may also account for the differences in concentration and polymerization of the hyaluronic acid.

Pathological effusions from human knee joints showed larger volumes than did the normal joints. The decrease in relative viscosity can, therefore, be considered as due in part to dilution. Effusions from osteoarthritis (degenerative) had the largest range and the highest average relative viscosity due both to the greater concentration of hyaluronic acid and the slightly higher mean polymerization.

The rheological findings in osteoarthritis reflect the pathological findings. In this disease destruction of synovial membrane is minimal or absent, inflammatory changes are secondary, and fre- quently there is villous hypertrophy. This hypertrophy makes possible increased production of hyaluronic acid even though the degree of polymerization is less than normal.

In rheumatoid arthritis the hyaluronic acid concentration, relative viscosity, intrinsic viscosity, and anomaly factor are the lowest for all types studied. No evidence of enzymatic degradation has been found to account for the low polymerization. Therefore, defective synthesis of the hyaluronic acid by the markedly inflamed, infiltrated, and partly eroded synovial membrane is a more likely explanation.

In chronic traumatic synovitis the relative viscosity and the hyaluronic acid concentrations are as low as in rheumatoid arthritis. However, the intrinsic viscosity and the anomaly factor are slightly higher. These findings indicate that the chronic irritation which follows minor trauma leads to marked dysfunction of the synovial membrane. If further investigation confirms these findings it will be of considerable diagnostic value, since objective laboratory, clinical and roentgenographic findings are often insufficient in traumatic synovitis.

For the differential diagnosis of the various types of joint diseases, a combination of the different rheologic findings in synovial effusions provides valuable, although limited, information. For example, synovial fluid with a high relative and anomalous viscosity is more likely to be associated with osteoarthritis than with rheumatoid arthritis or chronic traumatic synovitis.

Sundblad (3) tends to minimize the value of relative viscosity in the differential diagnosis of various types of arthritis. However, from our results it appears to be as valuable as any other single viscosity determination.

For clinical studies the simpler methods of determination of relative and anomalous viscosity which do not require hyaluronic acid determinations should be sufficient.

Viscosity measurement is only one aspect of a systematic examination of synovial effusions which includes cytological, chemical and bacteriological tests as described by Kling (1), Ropes and Bauer (2) and by Sundblad (3). Comprehensive study of the joint effusions combined with a thorough clinical, laboratory and roentgen study are necessary for an adequate differential diagnosis. 


\section{SUMMARY}

1. A simple capillary viscometer is described, which permits the determination of relative, intrinsic and anomalous viscosity of small volumes of synovial fluid.

2 . The various viscosity measurements were determined in synovial fluid from normal carpal joints of horses and cattle and from pathological effusions in human knee joints damaged by osteoarthritis, rheumatoid arthritis, and chronic traumatic synovitis.

3. Hyaluronic acid concentration on these fluids were carried out by a controlled turbidimetric method.

4. Normal synovial fluid from the carpus joint of cattle gave the highest average values for the relative viscosity, intrinsic viscosity and anomaly factor. The concentration of hyaluronic acid was high. The fluid from horses showed viscosity values which were lower than those found in cattle, and a lower hyaluronic acid concentration. The volume of fluid was small in both species compared to pathological human fluid.

5. In the pathological fluids, deviations were most marked in rheumatoid arthritis, less so in chronic traumatic synovitis, and least marked in osteoarthritis.

6. Interpretation of the factors responsible for the differences in viscous behavior are presented.

7. In conjunction with other tests the rheological behavior in normal and pathological fluids provides valuable information about the physiology and pathology of joints.

\section{ACKNOWLEDGMENTS}

We are indebted to Mr. G. Strasser, Wilson \& Co., Mr. Jon Edwards, Victory Packing Co., both of Los Angeles, for the animal joints, to Dr. Joseph Seifter of the Wyeth Institute of Applied Biochemistry for samples of sodium hyaluronate and streptococcal hyaluronidase, and to $\mathrm{Dr}$. Robert L. Craig of G. D. Searle \& Co. for testicular hyaluronidase.

\section{REFERENCES}

1. Kling, D. H., The Synovial Membrane and the Synovial Fluid, with Special Reference to Arthritis and Injuries of the Joints. Los Angeles, Med. Press, 1938.

2. Ropes, M. W., and Bauer, W., Synovial Fluid Changes in Joint Disease. Cambridge, Harvard University Press, 1953.
3. Sundblad, L., Studies on hyaluronic acid in synovial fluids. Acta Soc. Med. Upsalien., 1953, 58, 113.

4. Meyer, K., Smyth, E. M., and Dawson, M. H., The isolation of a mucopolysaccharide from synovial fluid. J. Biol. Chem., 1939, 128, 319.

5. Mann, F. D., A clinical viscometer. Am. J. Clin. Path., 1948, 18, 79.

6. Meyer, $\mathrm{K}$., The biological significance of hyaluronic acid and hyaluronidase. Physiol. Rev., 1947, 27, 335.

7. Schmith, K., and Faber, V., The turbidimetric method for determination of hyaluronidase. Scandinav. J. Clin. \& Lab. Invest., 1950, 2, 292.

8. Ragan, C., and Meyer, $K$., The hyaluronic acid of synovial fluid in rheumatoid arthritis. J. Clin. Invest., 1949, 28, 56.

9. Kass, E. H., and Seastone, C. V., The role of the mucoid polysaccharide (hyaluronic acid) in the virulence of group A hemolytic streptococci. J. Exper. Med., 1944, 79, 319.

10. Tolksdorf, S., McCready, M. H., McCullagh, D. R., and Schwenk, E., The turbidimetric assay of hyaluronidase. J. Lab. \& Clin. Med., 1949, 34, 74.

11. Kling, D. H., Levine, M. G., and Wise, S., Mucopolysaccharides in tissue cultures of human and mammalian synovial membrane. Proc. Soc. Exper. Biol. \& Med., 1955, 89, 261.

12. Schneider, J., Untersuchungen über die Viskosität menschlicher Synovia. Biochem. Ztschr., 1925, $160,322$.

13. Schulz, G. V., and Blaschke, F., Molecular-weight determinations on macromolecular materials. IX. An equation for the calculation of the viscosity number at very small concentrations. J. Prakt. Chem., 1941, 158, 130.

14. Fletcher, E., Jacobs, J. H., and Markham, R. L., Viscosity studies on hyaluronic acid of synovial fluid in rheumatoid arthritis and osteoarthritis. Clin. Sc., 1955, 14, 653.

15. Fessler, J. H., Ogston, A. G., and Stanier, J. E., Some properties of human and other synovial fluids. Biochem. J., 1954, 58, 656.

16. Johnston, J. P., The viscosity of normal and pathological human synovial fluids. Biochem. J., 1955, $59,633$.

17. Scott Blair, G. W., Williams, P. O., Fletcher, E. T. D., and Markham, R. L., On the flow of certain pathological human synovial effusions through narrow tubes. Biochem. J., 1954, 56, 504.

18. Ostwald, W., and Auerbach, R., Zur Messmethodik der Viskosimetrie bei variabler Fliessgeschwindigkeit und über ein neues Viskosimeter (UeberlaufViskosimeter). Kolloid Ztschr., 1927, 41, 56.

19. Zamboni, V. P., Die Strukturviskosität der Mucine und ein neuer Apparat zu ihrer Messung. Kolloid Ztschr., 1943, 102, 246. 